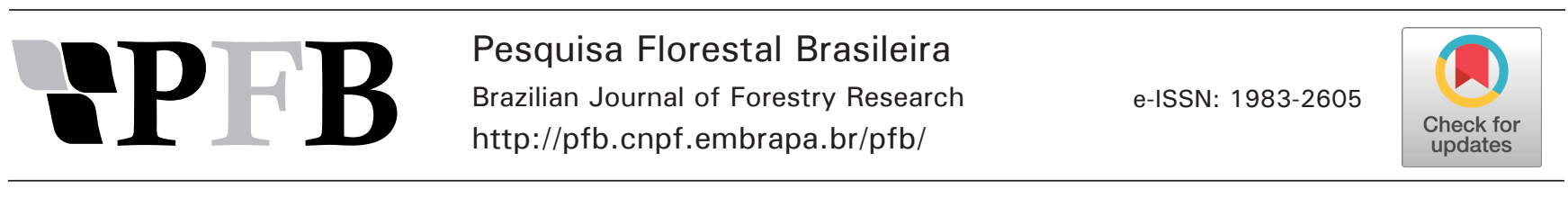

\title{
Redes neurais artificiais para estimar a densidade básica de madeiras do cerrado
}

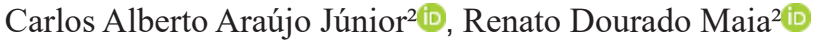 \\ ${ }^{1}$ Universidade Federal do Espírito Santo, Av. Governador Lindemberg; 316, CEP 29550-000, Jerônimo Monteiro, ES, Brasil \\ Universidade Federal de Minas Gerais, Av. Universitária, 1000, CEP 39404-547, Montes Claros, MG, Brasil \\ ${ }^{3}$ Universidade Federal do Paraná, Rua XV de Novembro, 1000, CEP 82590-300, Curitiba, PR, Brasil
}

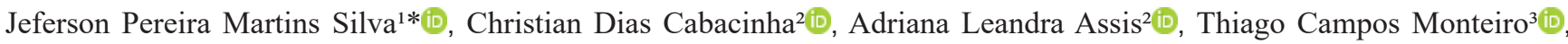

"Autor correspondente:

jefersonsb09@hotmail.com

Termos para indexação:

Inteligência artificial

Propriedades da madeira

Pilodyn

Index terms:

Artificial intelligence

Wood properties

Pilodyn

Histórico do artigo:

Recebido em 12/06/2018

Aceito em 11/12/2018

Publicado em 29/12/2018

doi: 10.4336/2018.pfb.38e201801656

\begin{abstract}
Resumo - A densidade básica da madeira é uma propriedade importante, pois está relacionada ao produto final nos diversos usos que a madeira possui. Porém, sua determinação demanda tempo e custos, o que justifica o emprego de técnicas mais refinadas para a sua estimação, como as redes neurais artificias (RNA). Objetivouse avaliar a utilização das RNA para estimar a densidade básica de espécies do cerrado sensu stricto com o uso do aparelho Pilodyn e variáveis dendrométricas. Para comparação dos resultados obtidos pelas RNA, foram ajustados modelos de regressão. A rede neural com melhor desempenho foi a que utilizou como variáveis de entrada a profundidade de penetração (Pilodyn), espécie e o diâmetro a $1,30 \mathrm{~m}$ do solo, apresentando $\mathrm{R}^{2}=0,72$ e com raiz do erro quadrado médio em porcentagem ( $\mathrm{RMSE}_{\%}$ ) $=5,69$. O modelo de regressão apresentou $\mathrm{R}^{2}=0,72$ e $\mathrm{RMSE}_{\%}=9,19$. É possível usar redes neurais artificiais para estimar a densidade básica da madeira das espécies do cerrado stricto sensu estudadas com resultados satisfatórios.
\end{abstract}

\section{Artificial neural network to estimate the basic density of cerrado wood}

\begin{abstract}
The basic density of wood is an important property because it is related to the final product in the various uses of wood. However, its determination demands time and costs, which justifies the use of more refined techniques for its estimation, such as artificial neural networks (ANN). Our objective was to evaluate the use of artificial neural networks to estimate the basic density of species of cerrado stricto sensu with the use of Pilodyn and dendrometric variables. To compare the results obtained by ANN, regression models were adjusted. The best performing neural network was the one that used as input variables the depth of penetration (Pilodyn), species and diameter at $1.3 \mathrm{~m}$ above ground level, presenting $\mathrm{R}^{2}=0.72$ and with root mean square error in percentage $(\mathrm{RMSE} \%)=5.69$. The regression model presented $\mathrm{R}^{2}=0.72$ and $\mathrm{RMSE} \%$ $=9.19$. The artificial neural networks can estimate the basic wood density of the studied species of cerrado stricto sensu with satisfactory results.
\end{abstract}

\section{Introdução}

A densidade básica da madeira é a relação entre a massa seca em estufa pelo respectivo volume da madeira acima do ponto de saturação das fibras, conforme NBR 11.941 (Associação Brasileira de Normas Técnicas, 2003). A sua determinação pode ser obtida diretamente por métodos destrutivos ou estimada por métodos não destrutivos. Para o primeiro caso, é necessário o abate das árvores para retirada de amostras, como discos ou cavacos. No segundo caso, sua determinação é através da retirada de pequenas amostras ou pequenas inserções feitas no tronco, sem a derrubada da árvore. 
A aplicação de métodos não destrutivos, a exemplo do que utiliza a espectroscopia de infravermelho próximo e técnica de resistografia, têm resultado em estimativas precisas para a densidade básica da madeira (Hein et al., 2009; Hein, 2012; Dias et al., 2017). Outro exemplo de método não destrutivo é o Pilodyn, que relaciona a densidade da madeira com a profundidade de penetração de uma agulha de aço inserida com força constante no tronco da árvore. Sua aplicação é interessante por proporcionar a obtenção de informações sem a derrubada da árvore.

Existem vários trabalhos a respeito da utilização Pilodyn para estimava de densidade básica de árvores de espécies plantadas, principalmente para os gêneros Eucalyptus (Vale et al., 1995; Thiersch et al., 2006; Couto et al., 2013) e Pinus (Cown \& Hutchison, 1983; Wang et al., 1999). Entretanto, não foi encontrada na literatura, sua aplicação em indivíduos arbóreos de espécies nativas do bioma Cerrado, motivo pelo qual seu emprego deve ser avaliado em tal situação. Além disso, a maioria dos trabalhos utiliza regressão linear simples, obtendo baixa correlação ou correlação não significativa entre a penetração do pino e a densidade básica (Bouffier et al., 2008), sendo importante a associação com técnicas computacionais mais refinadas, como as redes neurais artificiais (RNA), a fim de aumentar a precisão das estimativas.

Uma rede neural é um conjunto de unidades ou nós interligados, que tem processamento simples, cuja funcionalidade é baseada no neurônio biológico. Seu aprendizado é armazenado nas forças de conexão entre unidades, ou pesos sinápticos, obtidos por um processo de adaptação, a partir de um conjunto de padrões de treinamento (Haykin, 2001). Os nós que compõem as RNA calculam determinadas funções matemáticas, normalmente não lineares (Braga et al., 2007). São capazes de generalizar o conhecimento aprendido por meio de exemplos a um conjunto de dados desconhecidos (Haykin, 2001; Gorgens et al., 2009).

O uso das redes neurais tem se destacado nas pesquisas em Ciências Florestais, a exemplo dos estudos de Reis et al. (2018), para prognosticar a sobrevivência e a mortalidade de árvores na Amazônia; Vieira et al. (2018), para modelar o crescimento de árvores individuais; Leite et al. (2011), para estimação do diâmetro sem casca e diâmetro do cerne para árvores de Tectona grandis Linn; e Binoti et al. (2015), para prognose em nível de povoamento de clones de Eucalyptus sp. Além disso, as
RNA têm gerado maior acurácia com relação às técnicas estatísticas tradicionais (Aertsen et al., 2010; Özçelik et al., 2010, 2013; Görgens et al., 2015; Reis et al., 2018).

Por apresentar-se como um aproximador universal de funções, tal ferramenta demonstra ser uma boa alternativa para estimar a densidade básica de espécies nativas do cerrado, juntamente com o emprego do Pilodyn. Desta forma, objetivou-se avaliar a utilização das redes neurais artificiais para estimar a densidade básica de espécies do cerrado sensu stricto, a partir da profundidade de penetração do Pilodyn e variáveis dendrométricas e comparar os resultados obtidos com modelos de regressão.

\section{Material e métodos}

A área de coleta de dados está localizada a $16^{\circ} 41^{\prime} \mathrm{S}$ e 43501'W no município de Montes Claros, MG, e pertence ao Instituto de Ciências Agrárias da Universidade Federal de Minas Gerais. O clima do local é Aw sensu Köppen, tropical semiárido, quente e seco, com período de chuvas concentradas entre outubro e março. A precipitação anual é de $1.060 \mathrm{~mm}$ e a temperatura média anual é de $24,2{ }^{\circ} \mathrm{C}$ (Instituto Nacional de Meteorologia (INMET) (2018). A vegetação predominante é o cerrado sensu stricto.

Foram identificados botanicamente e medidos todos os indivíduos vivos, com diâmetro com casca a 1,3 m de altura do solo (DAP) igual ou superior a $3 \mathrm{~cm}$, excluindose os indivíduos das espécies protegidas pela legislação vigente. Obteve-se os valores de DAP, altura total (HT), altura do fuste comercial (HC) e resistência à penetração com o aparelho Pilodyn (PIL). Foram avaliados 334 indivíduos, distribuídos em 13 espécies (Tabelas 1 e 2).

Em todos os indivíduos selecionados, foram efetuadas, na posição do DAP sem casca, duas medições com o Pilodyn em lados opostos. Estas medições permitiram a obtenção de uma média aritmética da profundidade de penetração da agulha para cada indivíduo amostrado.

Para a determinação da densidade básica $\left(\mathrm{DB}_{\mathrm{d}}\right)$, as árvores foram abatidas e cinco discos de madeira, com aproximadamente $30 \mathrm{~mm}$ de espessura, foram retirados nas posições $0 \%, 25 \%, 50 \%, 75 \%$ e $100 \%$ da altura do fuste comercial. $\mathrm{A} \mathrm{DB}_{\mathrm{d}}$ dos discos $\left(\mathrm{g} . \mathrm{cm}^{-3}\right)$ foi obtida em laboratório, conforme NBR 11941 (Associação Brasileira de Normas Técnicas, 2003). Posteriormente, uma média aritmética da densidade básica da árvore foi calculada, utilizando as densidades obtidas em cada posição longitudinal da árvore. 
Tabela 1. Nome científico das espécies estudadas e a quantidade de indivíduos amostrados por espécie em cerrado sensu stricto, Montes Claros, MG.

Table 1. Scientific name of the studied species and the number of individuals sampled per species in cerrado stricto sensu, Montes Claros, MG.

\begin{tabular}{ccc}
\hline Espécie & quantidade & $\mathbf{n}^{\mathbf{0}}$ \\
\hline Luehea paniculata Mart. \& Zucc & 7 & $(1)$ \\
Terminalia fagifolia Mart. \& Zucc & 29 & $(2)$ \\
Copaifera langsdorffii Desf & 20 & $(3)$ \\
Maytenus sp. & 7 & $(4)$ \\
Heteropterys byrsonimifolia A. Juss & 120 & $(5)$ \\
Tocoyena formosa Cham. \& Schltdl & 19 & $(6)$ \\
Machaerium opacum Vog. & 55 & $(7)$ \\
Machaerium sp. & 7 & $(8)$ \\
Curatela americana L. & 22 & $(9)$ \\
Byrsonima heterophyla & 13 & $(10)$ \\
Combretum leprosum Mart & 12 & $(11)$ \\
Dalbergia brasiliensis Vog & 20 & $(12)$ \\
Magonia pubescens A. St \& Hil & 7 & $(13)$ \\
\hline
\end{tabular}

Os dados obtidos foram utilizados para treinar redes neurais artificiais do tipo perceptron de múltiplas camadas, conhecidas como MLP(Multilayer Perceptron). Considerou-se como variáveis de entrada a espécie, o DAP, a HT e a média da profundidade de penetração obtida com o Pilodyn (PIL) de cada indivíduo amostrado. Como variável de saída, considerou-se a DB.

As variáveis utilizadas para estimar a $\mathrm{DB}_{\mathrm{e}}$ foram classificadas em quantitativas independentes (DAP, HT e PIL), quantitativa dependente ( $\mathrm{DB}_{\mathrm{d}}$ ) e qualitativa ou categórica independente (espécies). As espécies foram transformadas em variáveis dummy, para treinamento e validação das MLP.

Para obtenção dos parâmetros das redes neurais artificiais, utilizou-se o Toolbox redes neurais artificiais do software MATLAB R2016a. As redes foram treinadas em quatro situações diferentes: (1) com todos os dados de entrada (MLP 1); (2) sem HT (MLP 2); (3) sem DAP (MLP 3); (4) sem as espécies (MLP 4).

Tabela 2. Número correspondente às espécies amostradas e valores mínimo, médio e máximo do diâmetro a 1,3 m do solo (DAP), da altura total (HT), da profundidade de penetração da agulha do aparelho Pilodyn (PIL) e da densidade básica da madeira (DB).

Table 2. Number corresponding to the species sampled and minimum, mean and maximum values of the diameter at $1.3 \mathrm{~m}$ from ground level (DBH), total height $(\mathrm{TH})$, penetration depth of the Pilodyn needle (PIL) and wood density (WD).

\begin{tabular}{|c|c|c|c|c|c|c|c|c|c|c|c|c|}
\hline \multirow{2}{*}{$\mathbf{n}^{\mathbf{o}}$} & \multicolumn{3}{|c|}{$\operatorname{DAP}(\mathbf{c m})$} & \multicolumn{3}{|c|}{ HT (m) } & \multicolumn{3}{|c|}{ PIL (mm) } & \multicolumn{3}{|c|}{ DB $\left(\mathrm{g} \mathrm{cm}^{-3}\right)$} \\
\hline & $\min$ & méd & máx & $\min$ & méd & máx & $\min$ & méd & máx & $\min$ & méd & máx \\
\hline (1) & 3,50 & 5,21 & 10,12 & 3,4 & 5,4 & 8,6 & 8,0 & 9,4 & 11,0 & 0,49 & 0,54 & 0,60 \\
\hline (2) & 3,34 & 5,21 & 9,33 & 3,3 & 4,7 & 6,7 & 5,6 & 9,3 & 11,5 & 0,56 & 0,62 & 0,70 \\
\hline (3) & 3,02 & 6,56 & 13,96 & 3,2 & 5,6 & 8,9 & 7,5 & 9,9 & 15,0 & 0,49 & 0,59 & 0,69 \\
\hline (4) & 3,18 & 5,79 & 8,21 & 3,8 & 4,9 & 5,9 & 6,0 & 9,1 & 12,5 & 0,61 & 0,64 & 0,68 \\
\hline (5) & 2,93 & 4,80 & 14,45 & 2,7 & 4,3 & 6,8 & 4,8 & 8,5 & 12,0 & 0,53 & 0,60 & 0,69 \\
\hline (6) & 3,34 & 4,56 & 6,94 & 2,7 & 4,2 & 6,5 & 6,5 & 8,5 & 14,0 & 0,57 & 0,63 & 0,73 \\
\hline (7) & 3,50 & 6,19 & 16,39 & 1,9 & 4,0 & 6,3 & 4,2 & 7,0 & 10,5 & 0,60 & 0,69 & 0,76 \\
\hline (8) & 3,92 & 5,45 & 7,32 & 3,9 & 6,8 & 8,8 & 6,5 & 8,0 & 9,0 & 0,58 & 0,62 & 0,67 \\
\hline (9) & 3,72 & 6,39 & 13,81 & 2,0 & 3,5 & 5,8 & 8,5 & 13,4 & 18,0 & 0,42 & 0,48 & 0,57 \\
\hline (10) & 3,25 & 6,43 & 12,11 & 3,2 & 4,7 & 6,2 & 5,5 & 10,3 & 14,5 & 0,49 & 0,57 & 0,70 \\
\hline (11) & 3,63 & 5,37 & 10,82 & 3,0 & 4,1 & 5,5 & 7,8 & 11,2 & 13,5 & 0,50 & 0,55 & 0,63 \\
\hline (12) & 3,02 & 5,69 & 10,89 & 3,1 & 5,0 & 7,3 & 7,0 & 10,1 & 12,5 & 0,49 & 0,56 & 0,61 \\
\hline (13) & 3,02 & 4,52 & 7,00 & 3,3 & 4,3 & 5,9 & 6,5 & 7,5 & 9,0 & 0,63 & 0,68 & 0,74 \\
\hline
\end{tabular}


Para que se possa utilizar MLP para estimar a $\mathrm{DB}_{\mathrm{e}}$ é necessário o ajuste dos parâmetros, que são os pesos sinápticos das conexões entre as unidades de processamento. Esses são ajustados em um processo iterativo, comumente chamado de aprendizagem ou treinamento (Braga et al., 2007). Os algoritmos de treinamento utilizados nesse estudo foram variações do método backpropagation, sendo eles o resilientpropagation e o Levenberg-Marquardat. Tais algoritmos foram criados para deixar o processo de convergência mais eficiente (Silva et al., 2010).

As redes Multilayer Perceptron são compostas de camada de entrada, onde as variáveis são apresentadas à rede; camadas intermediárias ou ocultas, onde é feito o processamento, e a camada de saída, onde o resultado é mostrado. A camada intermediária é responsável por identificar os padrões não-lineares dos dados, com a utilização de funções de ativação, sendo as mais comuns as funções linear, tangente hiperbólica, logística e a degrau (Braga et al., 2007). Nesse estudo foram utilizadas as funções tangente hiperbólica, logística e linear.

Treinou-se 100 redes neurais, alterando os algoritmos de treinamento, número de neurônios na camada oculta, que variou de 5 a 15, os tipos de funções de ativação na camada oculta e saída, até a definição da melhor arquitetura. Posteriormente, selecionaram-se as melhores redes para as três situações mencionadas anteriormente. O número de ciclos foi 1.000, e os dados foram separados por espécie de forma aleatória em $70 \%$ para treinamento e 30\% para validação. As melhores RNA foram selecionadas com base na correlação entre $\mathrm{a} \mathrm{DB}_{\mathrm{d}}$ e a $\mathrm{DB}_{\mathrm{e}}$ pelas redes, fornecidos pelo software nas fases de treinamento e validação.

Após a seleção da melhor RNA em cada situação, as estimativas da $\mathrm{DB}_{\mathrm{e}}$ foram avaliadas, considerando os seguintes critérios: gráficos da relação entre $\mathrm{a} \mathrm{DB}_{\mathrm{d}}$ e a $\mathrm{DB}_{\mathrm{e}}$, histograma de frequência dos erros percentuais, coeficiente de determinação $\left(\mathrm{R}^{2}\right)$ e a raiz quadrada do erro médio (RMSE - Root Mean Square Error) em porcentagem, sendo o erro obtido pela equação 1 .

$$
\operatorname{err} \circ(\%)=\frac{(\mathrm{Y}-\hat{\mathrm{Y}})}{\mathrm{Y}} 100
$$

Em que: $\mathrm{Y}=$ densidade básica determinada e $\hat{Y}=$ densidade básica estimada.
O coeficiente de determinação ( mede o grau de ajustamento dos dados (Campos \& Leite, 2017), sendo calculado pela equação 2 .

$$
R^{2}=1-\frac{\sum_{i=1}^{n}\left(Y_{i}-\hat{Y}_{i}\right)^{2}}{\sum_{i=1}^{n}\left(Y_{i}-\hat{Y}_{i}\right)^{2}}
$$

Em que: $Y_{i}=$ variável dependente observada; $\hat{Y}_{i}=$ variável dependente estimada; $\bar{Y}_{i}=$ média da variável dependente observada; $n=$ número de observações.

A raiz do erro quadrado médio foi obtida a partir da equação 3 (Mehtätalo et al., 2006).

$$
R M S E_{\%}=100 \frac{\sqrt{\frac{\sum_{i=1}^{n}\left(Y_{i}-\hat{Y}_{i}\right)^{2}}{n}}}{\overline{\mathrm{Y}}}
$$

Em que: $n=$ número total de dados; $\bar{Y}=$ média da densidade básica determinada; $Y_{i}=$ densidade básica determinada e, $\bar{Y}_{i}=$ densidade básica estimada.

A análise de regressão foi realizada para verificar a capacidade dos métodos tradicionais de estimativas e serviu de referência para comparar a alternativa proposta pela abordagem de redes neurais artificiais (Iliadis et al., 2013). Desse modo, foi realizada uma regressão linear múltipla para os mesmos conjuntos de dados, de acordo com cada situação estudada. As espécies foram transformadas em variáveis dummy, para fazer os ajustes dos modelos de regressão.

\section{Resultados}

Os valores dos coeficientes dos modelos de regressão bem como o resultado do teste t de Student, em nível de $5 \%$ de probabilidade de erro para as quatro situações analisadas estão apresentados na Tabela 3 .

As melhores arquiteturas da MLP para estimar a densidade básica nas três situações continham a função tangente hiperbólica na camada oculta, função linear na camada de saída, 12 neurônios na camada oculta para situação 1 e 2; e 7 neurônios para situação 3. Nas três situações o melhor algoritmo de treinamento foi o Levemberg-Marquardt. 
Tabela 3. Valores dos coeficientes da regressão e o resultado do teste $t$ de Student em nível significância de $95 \%$ para as distintas situações analisadas.

Table 3. Values of the regression coefficients and the Student $t$ test result at a significance level of $95 \%$ for the different situations analyzed.

\begin{tabular}{|c|c|c|c|c|}
\hline Variáveis & $\begin{array}{l}\text { Situação } \\
1\end{array}$ & $\begin{array}{l}\text { Situação } \\
\quad 2\end{array}$ & $\begin{array}{l}\text { Situação } \\
\mathbf{3}\end{array}$ & $\begin{array}{l}\text { Situação } \\
4\end{array}$ \\
\hline Intercepto & $0,6464^{*}$ & $0,6833^{*}$ & $0,6356^{*}$ & $0,7508^{*}$ \\
\hline $\begin{array}{l}\text { Luehea paniculata Mart. \& } \\
\text { Zucc }\end{array}$ & $-0,0758^{*}$ & $-0,1002^{*}$ & $-0,0713^{*}$ & - \\
\hline $\begin{array}{l}\text { Terminalia fagifolia Mart. } \\
\& \text { Zucc }\end{array}$ & $0,0038^{\mathrm{ns}}$ & $-0,0212^{\mathrm{ns}}$ & $0,0089^{\text {ns }}$ & - \\
\hline Copaifera langsdorffii Desf & $-0,0422^{*}$ & $-0,0664^{*}$ & $-0,0338^{\mathrm{ns}}$ & - \\
\hline Maytenus sp. & $0,0249^{\mathrm{ns}}$ & $0,0000^{\mathrm{ns}}$ & $0,0312^{\mathrm{ns}}$ & - \\
\hline $\begin{array}{l}\text { Heteropterys byrsonimifolia } \mathrm{A} \text {. } \\
\text { Juss }\end{array}$ & $\begin{array}{r}-0,0129 \\
\text { ns }\end{array}$ & $-0,0392^{*}$ & $-0,0072^{\mathrm{ns}}$ & - \\
\hline $\begin{array}{l}\text { Tocoyena formosa } \text { Cham. \& } \\
\text { Schltdl }\end{array}$ & $0,0157^{\mathrm{ns}}$ & $-0,0095^{\mathrm{ns}}$ & $0,0194^{\mathrm{ns}}$ & - \\
\hline Machaerium opacum Vog. & $0,0567^{*}$ & $0,0284^{\text {ns }}$ & $0,0673^{*}$ & - \\
\hline Machaerium sp. & $0,0000^{\mathrm{ns}}$ & $-0,0183^{\mathrm{ns}}$ & $0,0000^{\mathrm{ns}}$ & - \\
\hline Curatela americana $\mathrm{L}$ & $-0,1008^{*}$ & $-0,1314^{*}$ & $-0,0897^{*}$ & - \\
\hline Byrsonima heterophyla & $\begin{array}{r}-0,0342 \\
\text { ns }\end{array}$ & $-0,0610^{*}$ & $-0,0243^{\text {ns }}$ & - \\
\hline Combretum leprosum Mart & $\begin{array}{r}-0,0478 \\
\text { ns }\end{array}$ & $-0,0757^{*}$ & $-0,0412^{*}$ & - \\
\hline Dalbergia brasiliensis Vog & $-0,0536^{*}$ & $-0,0784^{*}$ & $-0,0489^{*}$ & - \\
\hline Magonia pubescens A.St \& Hil & $0,0643^{*}$ & $0,0391^{*}$ & $0,0689^{*}$ & - \\
\hline DAP & $0,0028^{*}$ & $0,0039^{*}$ & - & $0,0041^{*}$ \\
\hline HT & $0,0037^{\mathrm{ns}}$ & - & $0,0070^{*}$ & $0,0027^{\mathrm{ns}}$ \\
\hline PIL & $-0,0075^{*}$ & $-0,0075^{*}$ & $-0,0069^{*}$ & $-0,0206^{*}$ \\
\hline
\end{tabular}

Em que: * = significativo e ns= não significativo em nível de $95 \%$ de significância pelo teste $t$ de Student; Situação 1: regressão com todos os dados de entrada; situação 2: regressão sem HT; Situação 3: regressão sem DAP; Situação 4: regressão sem as espécies; DAP= diâmetro medido a 1,3 m do solo; HT= altura total.

As estatísticas dos treinamentos das RNA(Multilayer Perceptron - MLP 1, 2, 3 e 4) e ajustes dos modelos de regressão (Reg 1, 2, 3, e 4), obtiveram resultados semelhantes, com baixo RMSE\% e alto valor de $\mathrm{R}^{2}$ (Tabela 4). Os piores resultados foram observados na situação 4, em que foram retiradas as espécies do conjunto de treinamento e ajustes dos modelos.

As MLP apresentaram resultados superiores na validação dos dados para estimativa da densidade básica, comparada aos modelos de regressão para as quatro situações analisadas (Tabela 5). A MLP para a situação 1, que considerou como variáveis de entrada a espécie, diâmetro a 1,3 m do solo (DAP), altura total
(HT) e o Pilodyn, apresentou os melhores resultados de densidade básica estimada $\left(\mathrm{DB}_{\mathrm{e}}\right)$, com alto $\mathrm{R}^{2}$ e baixo RMSE\%, seguida das situações 2, 3 e 4. Observa-se que em todas as situações (Situação 1, 2, 3 e 4) em que utilizou a regressão no processamento dos dados (Tabela 5), apresentaram os piores resultados para o conjunto dos dados de validação em comparação com as redes neurais artificiais.

Tabela 4. Resultados das estatísticas RMSE, RMSE\% e $\mathrm{R}^{2}$ obtidos durante a fase de treinamento das Multilayer Perceptron (MLP) e ajustes dos modelos de regressão para as situações analisadas.

Table 4. Results of the RMSE, RMSE $\%$ and $\mathrm{R}^{2}$ statistics obtained during the Multilayer Perceptron (MLP) training phase and adjustments of the regression models for the situations analyzed.

\begin{tabular}{cccc}
\hline Avaliação & RMSE $\left(\mathbf{g ~ c m}^{-3}\right)$ & RMSE\% & $\mathbf{R}^{2}$ \\
\hline MLP 1 & 0,0346 & 5,73 & 0,74 \\
MLP 2 & 0,0334 & 5,53 & 0,72 \\
MLP 3 & 0,0333 & 5,51 & 0,72 \\
MLP 4 & 0,0453 & 7,50 & 0,49 \\
Reg 1 & 0,0328 & 5,43 & 0,72 \\
Reg 2 & 0,0330 & 5,46 & 0,72 \\
Reg 3 & 0,0332 & 5,49 & 0,72 \\
Reg 4 & 0,0464 & 7,67 & 0,45
\end{tabular}

Em que: $\mathrm{R}^{2}=$ coeficiente de determinação; $\mathrm{RMSE} \%$ = raiz do erro quadrado médio em porcentagem.

Tabela 5. Resultados das estatísticas RMSE, RMSE\% e $\mathrm{R}^{2}$ obtidos na validação dos dados para estimativa da densidade básica, usando as MLP e as equações de regressão para as quatro situações analisadas.

Table 5. Results of RMSE, RMSE $\%$ and $\mathrm{R}^{2}$ statistics obtained in the validation of the data for basic density estimation using MLP and regression equations for the four analyzed situations.

\begin{tabular}{cccc}
\hline Avaliação & RMSE $\left(\mathbf{g ~ c m}^{-3}\right)$ & RMSE\% & $\mathbf{R}^{2}$ \\
\hline MLP 1 & 0,0346 & 5,69 & 0,72 \\
MLP 2 & 0,0401 & 6,59 & 0,67 \\
MLP 3 & 0,0420 & 6,91 & 0,66 \\
MLP 4 & 0,0424 & 6,98 & 0,61 \\
Reg 1 & 0,0559 & 9,19 & 0,71 \\
Reg 2 & 0,1039 & 17,09 & 0,59 \\
Reg 3 & 0,0817 & 13,43 & 0,64 \\
Reg 4 & 0,0556 & 9,15 & 0,52 \\
\hline
\end{tabular}

Em que: $\mathrm{MLP}=$ Multilayer Perceptron; $\mathrm{R}^{2}=$ coeficiente de determinação; $\mathrm{RMSE} \%$ = raiz do erro quadrado médio em porcentagem. 
Na Figura 1 são apresentadas as relações entre as densidades básicas determinada $\left(\mathrm{DB}_{\mathrm{d}}\right)$ e estimada $\left(\mathrm{DB}_{\mathrm{e}}\right)$ que utilizaram a MLP (Figuras 1A; 1D; $1 \mathrm{G}$ e $1 \mathrm{~J}$ ) na validação dos dados apresentaram inclinação próxima aos $45^{\circ}$, demonstrando existir uma forte correlação positiva entre a $\mathrm{DB}_{\mathrm{d}}$ e DB. A MLP 4 (Figura 1J) apresentou menor aproximação dos pontos ao longo da reta, quando comparada à apresentação das outras relações.

A dispersão residual em porcentagem da MLP1 e MLP 2 (Figuras 1B e 1E), aparentemente, não apresentaram tendenciosidades, com os erros próximos à linha de erro zero e bem distribuídos. Os histogramas de erro em porcentagem (Figuras $1 \mathrm{C}$ e $1 \mathrm{~F}$ ) ficaram próximos à curva normal, com intervalos de $\pm 15 \%$.

A representação da MLP 3 (Figura 1H), apresentaram tendenciosidade em subestimar os valores de menores DB e superestimar os de maiores valores, com erros variando entre $+10 \%$ a $-30 \%$ (Figura 1 I).

A MLP 4 apresentou maior dispersão dos pontos (Figura $1 \mathrm{~K}$ ), com amplitude de erro de $\pm 20 \%$.
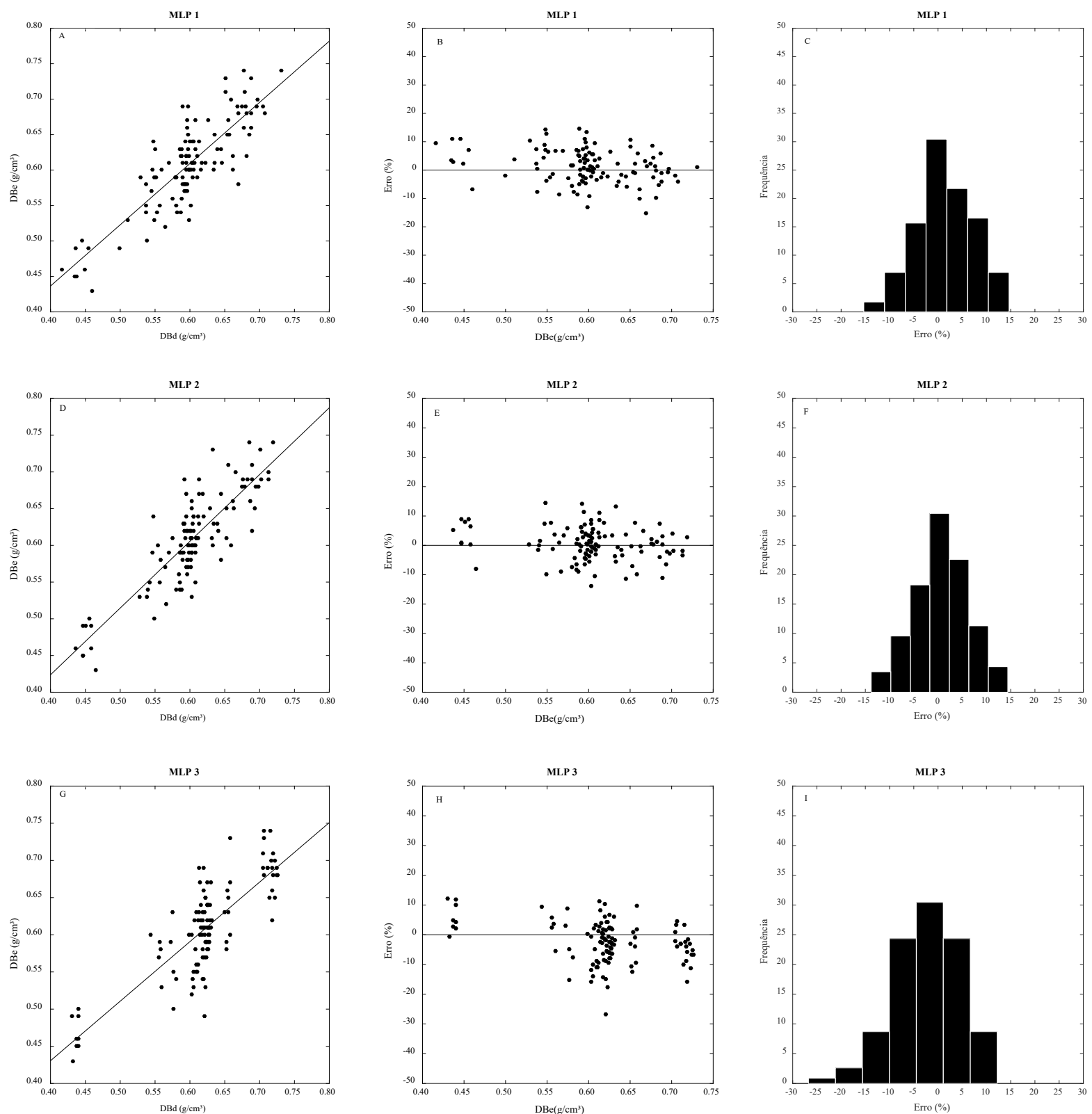

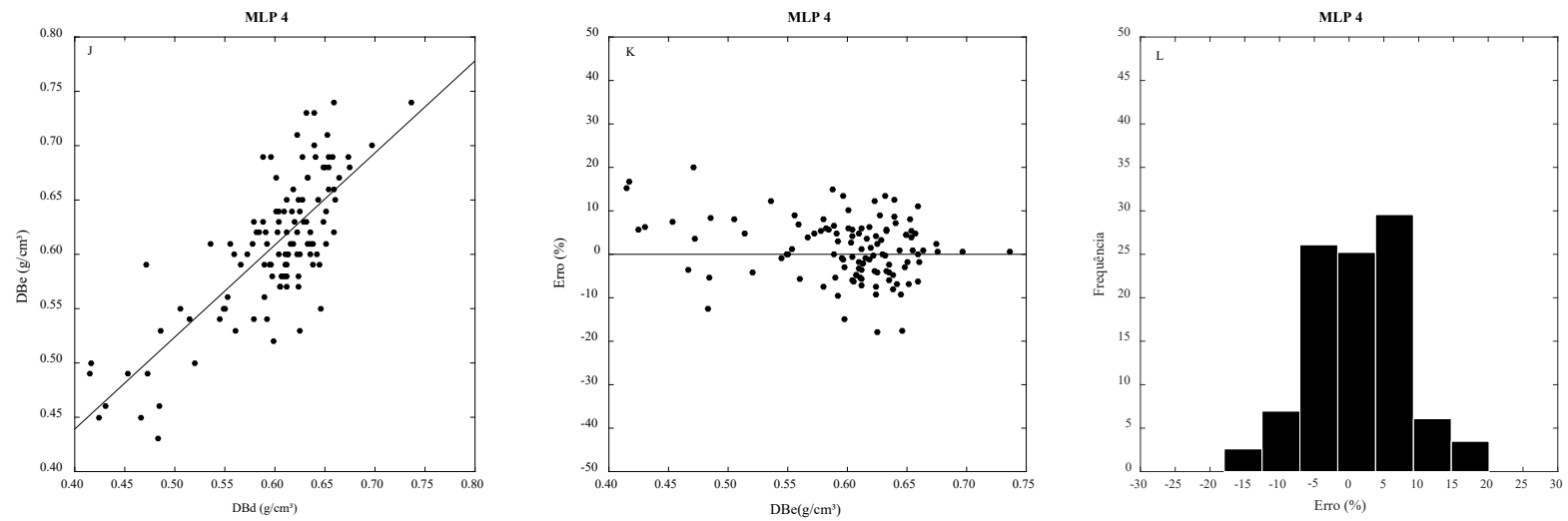

Figura 1. Relação entre a densidade básica determinada $\left(\mathrm{DB}_{\mathrm{d}}\right)$ e a estimada $\left(\mathrm{DB}_{\mathrm{e}}\right)$, dispersão residual em porcentagem em função da $\mathrm{DB}_{\mathrm{e}}$ e histograma de frequências relativas de erros em porcentagem para os dados de validação estimados pelas Multilayer Perceptron (MLP) 1, 2, 3 e 4.

Figure 1. Relationship between determinate $\left(\mathrm{DB}_{\mathrm{d}}\right)$ and estimated $\left(\mathrm{DB}_{\mathrm{e}}\right)$ basic density, residual dispersion in percentage as a function of $\mathrm{DB}_{\mathrm{e}}$ and histogram of relative frequency of errors in percentage for validation of data estimated by Multilayer Perceptron (MLP) 1, 2, 3 and 4.

Na Figura 2 são apresentados os resultados que utilizaram a regressão na validação dos dados para estimar a densidade básica, demonstrando relação positiva entre $a_{D_{d}}$ e $\mathrm{DB}_{\mathrm{e}}$ (Figuras 2A; 2D; 2G e $2 \mathrm{~J}$ ). Porém, apenas a Reg 1 não apresentou tendenciosidade evidente, com valores de erros próximos à linha zero e bem distribuídos (Figura 2B) e amplitude variando $\pm 15 \%$ (Figura 3C). As Reg 1 e Reg 2 apresentaram tendenciosidade, superestimando valores de densidade básica (Figuras $2 \mathrm{E}$ e $2 \mathrm{H}$ ), com erro variando de $-30 \%$ a $15 \%$ (Figuras $2 \mathrm{~F}$ e $2 \mathrm{I}$ ).
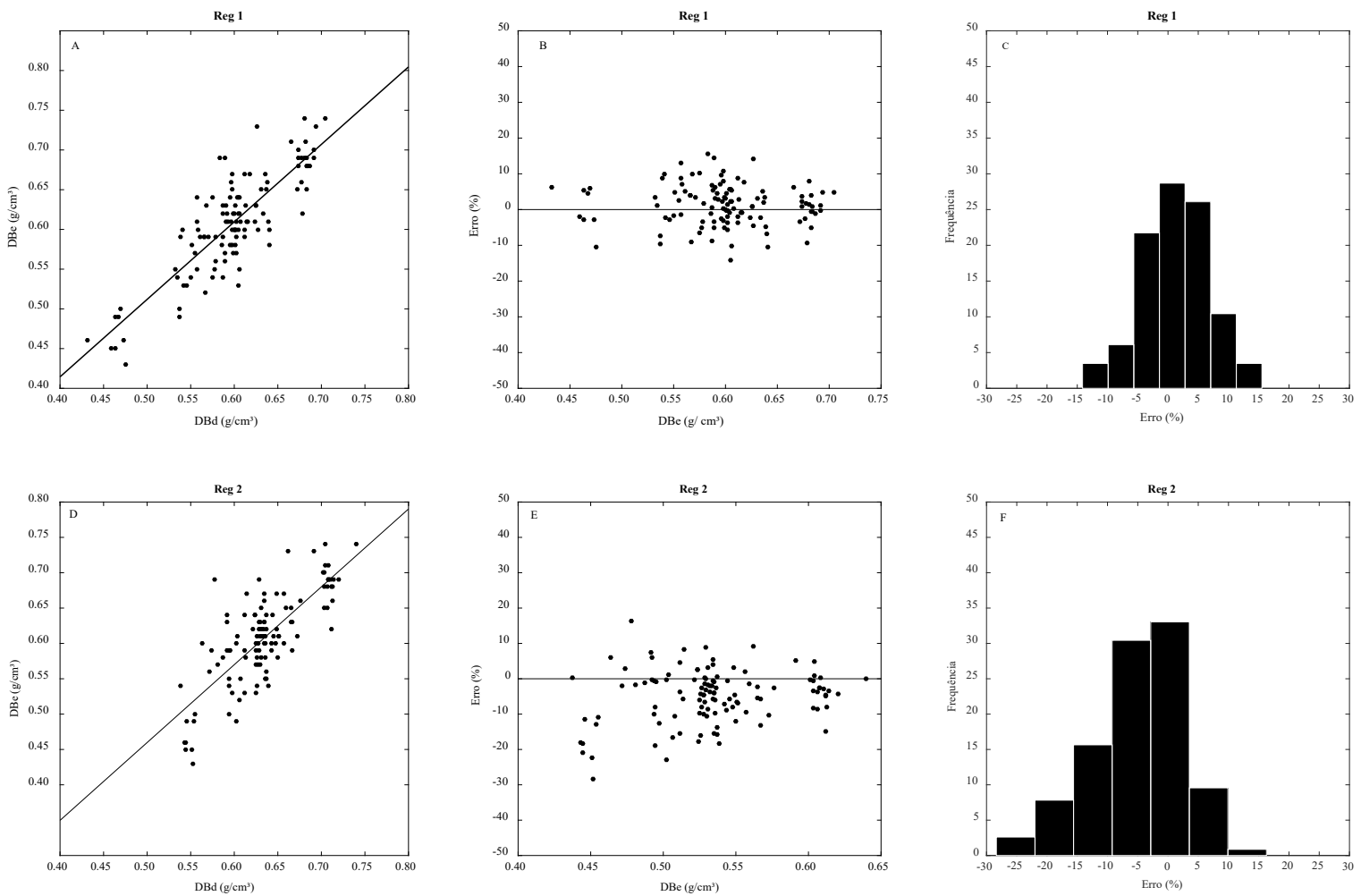

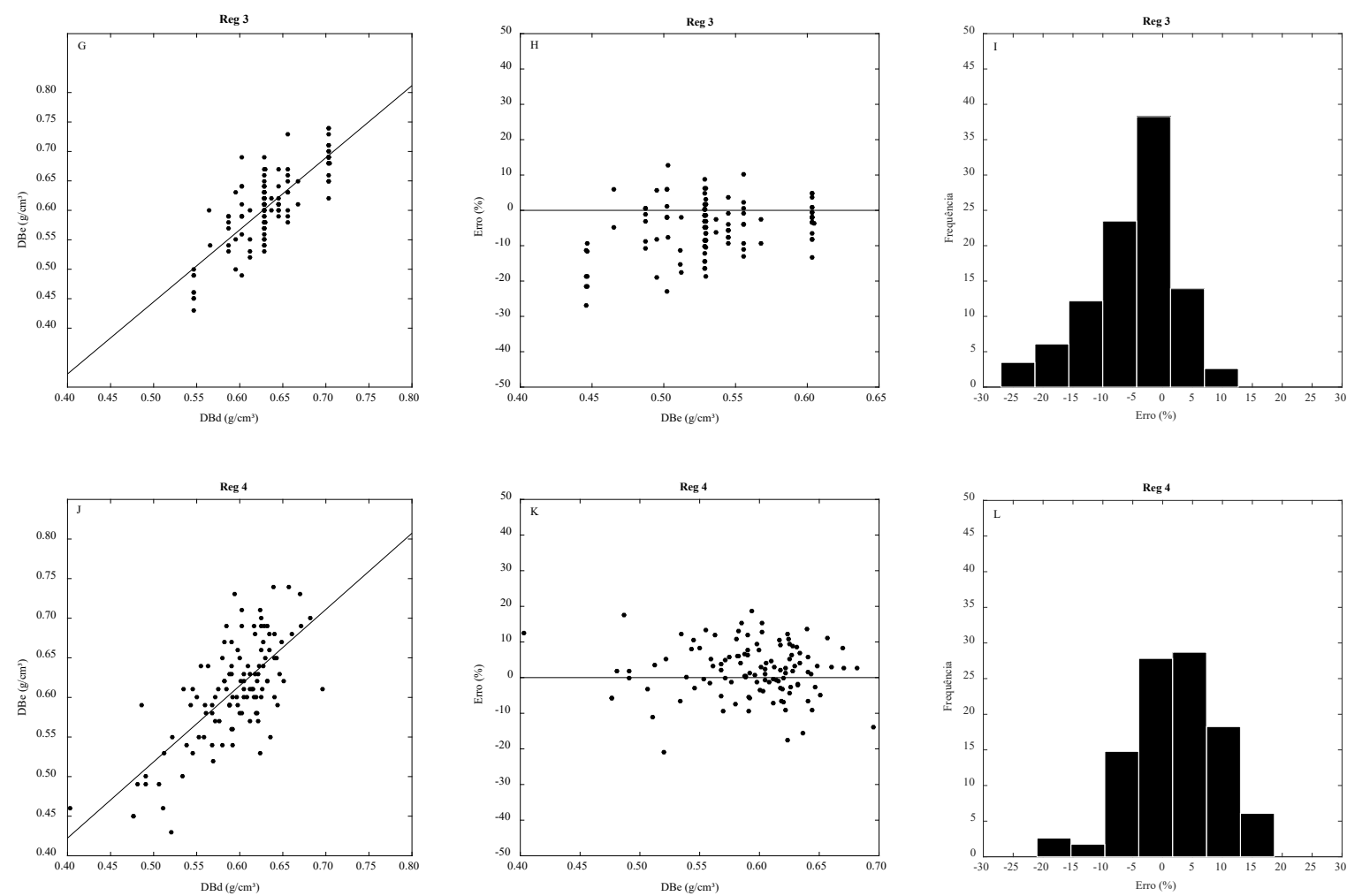

Figura 2. Relação entre a densidade básica determinada $\left(\mathrm{DB}_{\mathrm{d}}\right)$ e a estimada $\left(\mathrm{DB}_{\mathrm{e}}\right)$, dispersão residual em porcentagem em função da $\mathrm{DB}_{\mathrm{e}}$ e e histograma de frequências relativas de erros em porcentagem para os dados de validação estimados pelos modelos de regressão - Reg 1, 2, 3 e 4.

Figure 2. Relationship between determinate $\left(\mathrm{DB}_{\mathrm{d}}\right)$ and estimated $\left(\mathrm{DB}_{\mathrm{e}}\right)$ basic density, residual dispersion in percentage as a function of $\mathrm{DB}_{\mathrm{e}}$ and relative frequency histogram of errors in percentage for the validation data estimated by the regression models - Reg 1, 2, 3 and 4.

\section{Discussão}

As redes neurais artificiais mostraram superioridade ao método de regressão na estimativa de densidade básica de espécies do cerrado sensu stricto avaliadas nesse trabalho, principalmente as que utilizaram como variáveis de entrada as espécies, o diâmetro a 1,3 $\mathrm{m}$ do solo (DAP), a altura total (HT) e o Pilodyn. Isso pode ser observado também na Tabela 3, comparando os valores de raiz do erro quadrado médio em porcentagem (RMSE\%) das Multilayer Perceptron (MLP) que variaram entre 5,69 e 6,98 com os valores de RMSE\% das equações de regressão, que variaram de 9,15 a 17,09.

$\mathrm{O}$ uso de redes neurais e a profundidade de penetração da agulha do Pilodyn forneceram resultados satisfatórios neste estudo, que é um ponto relevante para difundir o uso deste equipamento. Bouffier et al. (2008) apresentam uma revisão de literatura com baixas correlações entre a densidade da madeira de coníferas e a profundidade de penetração da agulha do Pilodyn, onde os autores recomendaram a não utilização desta ferramenta para estimar a densidade básica da madeira em árvores deste grupo. É importante ressaltar que os autores fazem o relato apenas para madeira de coníferas. No entanto, os resultados obtidos nesse estudo (Tabelas 4 e 5) com o uso de redes neurais para estimar a densidade básica da madeira de espécies nativas do cerrado brasileiro demonstraram bons resultados utilizando a ferramenta Pilodyn. Mesmo os resultados das quatro regressões apresentaram estatísticas satisfatórias quando comparadas com a literatura. A exemplo, em estudo de com densidade básica da madeira de espécies arbóreas de Cerradão. Silva et al. (2015) encontraram erro padrão da estimativa em percentagem que variaram de $4,41 \%$ a $5,16 \%$.

Em estudos com madeiras de coníferas e folhosas as correlações entre a penetração da agulha e a densidade da madeira com o uso de regressão apresentam resultados 
satisfatórios. Cown (1978) encontrou - 0,97 de correlação entre a profundidade de penetração da agulha e a densidade da madeira de árvores de Pinus radiata com 10 anos. Em outro estudo, Cown \& Hutchison (1983) encontraram - 0,80 de correlação entre a profundidade de penetração da agulha e a densidade da madeira em tábuas de Pinus radiata com 15 e 25 anos. Em folhosas é possível encontrar estudos com coeficiente de correlação superiores aos obtidos nas regressões Reg 1, Reg 2, Reg 3 e Reg 4 deste estudo (Tabelas 4 e 5). Thiersch et al. (2006) trabalharam com densidade básica de dois clones de Eucalyptus sp. e encontraram, com a utilização da regressão e de variáveis dendrométricas e de profundidade de penetração com o aparelho Pilodyn, coeficientes de correlação de 0,94 e 0,96.

A superioridade das redes neurais para estimar a densidade básica é diminuída quando se retira o DAP, a HT e as espécies como variáveis de entrada, conforme observado nas situações 2, 3 e 4 da Tabela 5 e também na Figura 1. Isso pode ser atribuído ao fato de a densidade básica variar em função dessas variáveis.

A densidade básica é um parâmetro que apresenta variação entre espécies (Vale et al., 2010), dentro da espécie e dentro da própria árvore (Henry et al., 2010). A variação de densidade da madeira entre a região da medula e a região da casca é superior à variação da região da base para o topo da árvore (Cruz et al., 2003). As variações são significativas e alteram conforme a amostragem na árvore (Pádua et al., 2015). Estudos envolvendo a variação da densidade básica da madeira do gênero Eucalyptus e de outras espécies mostram um aumento no sentido medula-casca e uma diminuição no sentido base-topo (Cruz et al., 2003). Dessa forma, tanto a HT como o DAP podem alterar a estimativa da densidade básica da madeira.

Iliadis et al. (2013) encontraram os melhores resultados para estimativa de densidade básica da madeira utilizando a função de transferência tangente hiperbólica na camada oculta com 12 neurônios. Eles avaliaram a aplicação das redes neurais artificiais para estimar a densidade básica da madeira de quatro espécies de coníferas. Concluíram que as redes neurais artificiais conseguem utilizar com maior precisão as variáveis altura, DAP, densidade, volume e velocidade acústica, para estimar a densidade básica da madeira, comparada ao método de regressão. Os autores encontraram valores de coeficiente de correlação de 0,71 e 0,72 com a utilização das redes neurais e 0,48 com a utilização da regressão linear múltipla.

A utilização de redes neurais artificiais tem se demonstrado uma alternativa vantajosa, quando comparada às técnicas de regressão no manejo dos recursos florestais (Binoti et al., 2013). Dessa forma, também podem ser aplicadas para estimar a densidade básica a partir das variáveis obtidas no inventário florestal (Leite et al., 2016). Os resultados apresentados nesse estudo demonstram que é possível estimar a densidade básica de espécies do cerrado sensu stricto com precisão, utilizando-se redes neurais, a partir de variáveis que são normalmente medidas durante o inventário florestal (diâmetro e altura total) e a profundidade de penetração medida na altura do DAP obtida com o Pilodyn.

\section{Conclusão}

A utilização das redes neurais artificiais (RNA) para estimação da densidade básica da madeira de espécies do cerrado sensu stricto, fornece resultados satisfatórios, indicando a possibilidade do seu emprego para estimativa das mesmas.

Os resultados estatísticos obtidos pelas redes neurais artificiais e pelos modelos de regressão foram próximos.

A RNA que não incluiu a altura das árvores como variável de entrada apresentou resultados superiores, quando comparada às demais situações analisadas.

\section{Agradecimentos}

Os autores agradecem ao Conselho Nacional de Desenvolvimento Científico e Tecnológico (CNPq) pelo financiamento dessa pesquisa.

\section{Referências}

Aertsen, W. et al. Comparison and ranking of different modelling techniques for prediction of site index in Mediterranean mountain forests. Ecolological Modelling, v. 221, n. 8, p. 1119-1130, 2010. DOI: 10.1016/j.ecolmodel.2010.01.007.

Associação Brasileira de Normas Técnicas. NBR-11941: madeira: determinação da densidade básica. Rio de Janeiro, 2003.

Binoti, M. L. M. S. et al. Aplicação de redes neurais artificiais para estimação da altura de povoamentos equiâneos de eucalipto. Revista Árvore, v. 37, n. 4, p. 639-645, 2013. DOI: 10.1590/S010067622013000400007.

Binoti, M. L. M. S. et al. Prognose em nível de povoamento de clones de eucalipto empregando redes neurais artificiais. Cerne, v. 21, n. 1, p. $97-105$, 2015. DOI: 10.1590/01047760201521011153. 
Bouffier, L. et al. Can wood density be efficiently selected at early stage in maritime pine (Pinus pinaster Ait.) Annals of Forest Science, v. 65, n. 1, p. 1-8, 2008. DOI: 10.1051/forest:2007078.

Braga, A. P. et al. (Ed.). Redes neurais artificiais: teoria e aplicações. Rio de Janeiro, RJ: LTC, 2007. 226 p.

Campos, J. C. C. \& Leite, H. G. (Ed.). Mensuração florestal: perguntas e respostas. Viçosa, MG: UFV, 2017. 636 p.

Couto, A. M. et al. Modelagem da densidade básica da madeira de Eucalyptus grandis e Eucalyptus urophylla por métodos não destrutivos. Cerne, v. 19, n. 1, p. 27-34, 2013.

Cown, D. J. Comparasion of the pilodyn and torsiometer methods for the rapid assessment of wood density in living trees. New Zealand Journal of Forestry Science, v. 8, n. 3, p. 384-391, 1978.

Cown, D. J. \& Hutchison, J. D. Wood density as an indicator of the bending properties of Pinus radiata poles. New Zealand Journal of Forestry Science, v. 13, n. 1, p. 87-99, 1983.

Cruz, C. R. et al. Variação dentro das árvores e entre clones das propriedades físicas e mecânicas da madeira de híbridos de Eucalyptus. Scientia Forestalis, v. 64, n. 34, p. 33-47, 2003.

Dias, D. C. et al. Uso da técnica de resistografia e de variáveis dendrométricas na modelagem da densidade básica de povoamentos clonais de Eucalyptus. Ciência Florestal, v. 27, n. 2, p. 609-619, 2017.

Görgens, E. B. et al. A performance comparison of machine learning methods to estimate the fast-growing forest plantation yield based on laser scanning metrics. Computers and Electronics in Agriculture, v. 116, n. 1, p. 221-227, 2015. DOI: 10.1016/j.compag.2015.07.004.

Gorgens, E. B. et al. Estimação do volume de árvores utilizando redes neurais artificiais. Revista Árvore, v. 33, n. 6, p. 1141-1147, 2009. DOI: 10.1590/S0100-67622009000600016.

Haykin, S. (Ed.). Redes neurais: princípios e prática. Porto Alegre: Bookman, 2001. 900 p.

Hein, P. R. G. Estimating shrinkage microfibril angle and density of Eucalyptus wood using near infrared spectroscopy. Journal of Near Infrared Spectroscopy, v. 20, p. 427-436, 2012.

Hein, P. R. G. et al. Robustness of models based on near infrared spectra to predict the basic density in Eucalyptus urophylla Wood. Journal of Near Infrared Spectroscopy, v. 17, n. 1, p. 141-150, 2009. DOI: $10.1255 /$ jnirs.833.

Henry, M. et al. Wood density, phytomass variations within and among trees, and allometric equations in a tropical rainforest of Africa. Forest Ecology and Management, v. 260, n. 8, p. 13751388, 2010.

Iliadis, L. et al. Predicting Douglas-fir wood density by artificial neural networks (ANN) based on progeny testing information. Holzforschung, v. 67 , n. 7 , p. 71-777, 2013. DOI: $10.1515 /$ hf2012-0132.
Instituto Nacional de Meteorologia. Banco de dados meteorológicos para ensino e pesquisa. Disponível em: < http://www.inmet.gov.br/ portal/>. Acesso em: 21 maio 2018.

Leite, H. G. et al. Estimation of inside-bark diameter and heartwood diameter for Tectona grandis Linn. trees using artificial neural networks. European Journal of Forest Research, v. 130, p. 263269, 2011. DOI: 10.1007/s10342-010-0427-7.

Leite, H. G. et al. Redes neurais artificiais para a estimação da densidade básica da madeira. Scientia Forestalis, v. 44, n. 1 p. 149-154, 2016.

Mehtätalo, L. et al. The Use of quantile trees in the prediction of the diameter distribution of a stand. Silva Fennica, v. 40, n. 3, p. 501-516, 2006.

Özçelik, R. et al. Estimating crimean juniper tree height using nonlinear regression and artificial neural network models. Forest Ecology Management, v. 306, n. 1 p. 52-60, 2013. DOI: 10.1016/j. foreco.2013.06.009.

Özçelik, R. et al. Estimating tree bole volume using artificial neural network models for four species in Turkey. Journal os Envionmental Management, v. 91, n. 3, p. 742-753, 2010. DOI: 10.1016/j.jenvman.2009.10.002.

Pádua, F. A. et al. Tamanho amostral para a estimativa da densidade básica em um clone híbrido de Eucalyptus sp. Revista do Instituto Florestal, v. 27, n. 1, p. 41-47, 2015. DOI: 10.4322/rif.2015.004.

Reis, L. P. et al. Estimation of mortality and survival of individual trees after harvesting wood using artificial neural networks in the amazon rain forest. Ecological Engineering, v. 112, n. 1, p. 140-147, 2018. DOI: 10.1016/j.ecoleng.2017.12.014.

Silva, C. J. et al. Densidade básica da madeira de espécies arbóreas de Cedão no estado do Tocantins. Pesquisa Florestal Brasileira, v. 35, n. 82, p. 63-75. DOI: 10.4336/2015.pfb.35.82.822.

Silva, I. N. et al. (Ed.). Redes neurais artificiais: para engenharia e ciências aplicadas. São Paulo, SP: Artliber, 2010. 393 p.

Thiersch, C. R. et al. O uso de modelos matemáticos na estimativa da densidade básica da madeira em plantios de clones de Eucalyptus sp. Cerne, v. 12, n. 3, p. 264-278, 2006.

Vale, A. T. et al. Densidade básica média em função da profundidade de penetração do pino do Pilodyn e da classe diamétrica e variação axial da densidade básica em Eucalyptus grandis Hill ex Maiden. Revista Árvore, v. 19, n. 1, p. 80-91, 1995.

Vale, A. T. et al. Relações entre propriedades químicas, físicas e energéticas da madeira de cinco espécies de Cerrado. Ciência Florestal, v. 20, n. 1, p 137-145, 2010.

Vieira, G. C. et al. Prognoses of diameter and height of trees of eucalyptus using artificial intelligence. Science of The Total Environment, v. 619, n. 1, p. 1473-1481, 2018. DOI: 10.1016/j. scitotenv.2017.11.138.

Wang, T. et al. Selection for height growth and Pilodyn pin penetration in lodgepole pine: effects on growth traits, wood properties, and their relationships. Canadian Journal of Forest Research, v. 29, n. 1, p. 434-445, 1999. DOI: 10.1139/x99-012. 